EGU2020-1887

https://doi.org/10.5194/egusphere-egu2020-1887

EGU General Assembly 2020

(c) Author(s) 2020. This work is distributed under

the Creative Commons Attribution 4.0 License.

\title{
Mapping Subsurface Drainage in Agricultural Areas Using a Frequency-Domain Ground Penetrating Radar
}

\author{
Triven Koganti ${ }^{1}$, Ellen Van De Vijver ${ }^{2}$, Barry J. Allred ${ }^{3}$, Mogens H. Greve ${ }^{1}$, Jørgen Ringgaard ${ }^{4}$, and Bo \\ V. Iversen \\ ${ }^{1}$ Department of Agroecology, Aarhus University, Tjele, Denmark \\ ${ }^{2}$ Research Group Soil Spatial Inventory Techniques, Department of Environment, Ghent University, Ghent, Belgium \\ ${ }^{3}$ USDA/ARS Soil Drainage Research Unit, Columbus, Ohio, U.S.A \\ ${ }^{4}$ Rambøll, Copenhagen, Denmark
}

Artificial subsurface drainage systems are installed in agricultural areas to remove excess water and convert poorly naturally drained soils into productive cropland. Some of the most productive agricultural regions in the world are a result of subsurface drainage practices. Drain lines provide a shortened pathway for the release of nutrients and pesticides into the environment, which presents a potentially increased risk for eutrophication and contamination of surface water bodies. Knowledge of drain line locations is often lacking. This complicates the understanding of the local hydrology and solute dynamics and the consequent planning of mitigation strategies such as constructed wetlands, saturated buffers, bioreactors, and nitrate and phosphate filters. In addition, accurate knowledge of the existing subsurface drainage system is required in designing the installation of a new set of drain lines to enhance soil water removal efficiency. The traditional methods of drainage mapping involve the use of tile probes and trenching equipment which are time-consuming, tiresome, and invasive, thereby carrying an inherent risk of damaging the drain pipes. Non-invasive geophysical sensors provide a potential alternative solution to the problem. Previous research has focused on the use of time-domain ground penetrating radar (GPR) with variable success depending on local soil and hydrological conditions and the center frequency of the specific equipment used. For example, $250 \mathrm{MHz}$ antennas proved to be more suitable for drain line mapping. Recent technological advancements enabled the collection of high-resolution spatially exhaustive data. In this study, we present the use of a stepped-frequency continuous wave (SFCW) 3D-GPR (GeoScope Mk IV 3D-Radar with DXG1820 antenna array) mounted in a motorized survey configuration with real-time georeferencing for subsurface drainage mapping. The 3D-GPR system offers more flexibility for application to different (sub)surface conditions due to the coverage of wide frequency bandwidth (60-3000 MHz). In addition, the wide array swathe of the antenna array ( $1.5 \mathrm{~m}$ covered by 20 measurement channels) enables effective coverage of three-dimensional (3D) space. The surveys were performed on twelve different study sites with various soil types with textures ranging from sand to clay till. While we achieved good success in finding the drainage pipes at five sites with sandy, sandy loam, loamy sand and organic topsoils, the results at the other seven sites with more clay-rich soils were less successful. The high attenuation of electromagnetic waves in highly conductive clay-rich soils, which limits the 
penetration depth of the 3D-GPR system, can explain our findings obtained in this research. 